\title{
Solution and Gas-Phase H/D Exchange of Protein-Small-Molecule Complexes: Cex and Its Inhibitors
}

\author{
Yang Kang, ${ }^{1}$ Peran Terrier, ${ }^{2}$ Chuanfan Ding, ${ }^{3}$ D. J. Douglas ${ }^{1}$ \\ ${ }^{1}$ Department of Chemistry, University of British Columbia, 2036 Main Mall, Vancouver, BC, V6T 1Z1, Canada \\ ${ }^{2}$ Adocia, 115 Avenue Lacassagne, 69003 Lyon, France \\ ${ }^{3}$ Department of Chemistry, Fudan University, Shanghai, China
}

\begin{abstract}
The properties of noncovalent complexes of the enzyme exo-1,4- $\beta$-D-glycanase ("Cex") with three aza-sugar inhibitors, deoxynojirimycin $\left(\mathrm{X}_{2} \mathrm{DNJ}\right)$, isofagomine lactam $\left(\mathrm{X}_{2} \mathrm{IL}\right)$, and isofagomine $\left(\mathrm{X}_{2} \mathrm{IF}\right)$, have been studied with solution and gas-phase hydrogen deuterium exchange $(\mathrm{H} / \mathrm{Dx})$ and measurements of collision cross sections of gas-phase ions. In solution, complexes have lower H/Dx levels than free Cex because binding the inhibitors blocks some sites from $\mathrm{H} / \mathrm{Dx}$ and reduces fluctuations of the protein. In mass spectra of complexes, abundant Cex ions are seen, which mostly are formed by dissociation of complexes in the ion sampling interface. Both complex ions and Cex ions formed from a solution containing complexes have lower cross sections than Cex ions from a solution of Cex alone. This suggests the Cex ions formed by dissociation "remember" their solution conformations. For a given charge, ions of the complexes have greater gas-phase $H / D x$ levels than ions of Cex. Unlike cross sections, $H / D x$ levels of the complexes do not correlate with the relative gas-phase binding strengths measured by MS/MS. Cex ions from solutions with or without inhibitors, which have different cross sections, show the same $\mathrm{H} / \mathrm{Dx}$ level after $15 \mathrm{~s}$, indicating the ions may fold or unfold on the seconds time scale of the H/Dx experiment. Thus, cross sections show that complexes have more compact conformations than free protein ions on the time scale of ca. $1 \mathrm{~ms}$. The gas-phase H/Dx measurements show that at least some complexes retain different conformations from the Cex ions on a time scale of seconds.
\end{abstract}

Key words: Noncovalent complex, Cross section, Hydrogen deuterium exchange, Binding energy, Trapped ions

\section{Introduction}

S tudies by mass spectrometry (MS) of the noncovalent binding of proteins to other proteins or small molecules continue to be of interest, with the potential for a better understanding of noncovalent binding properties, both in solution and in gas-phase ions. The "soft" ionization technique, electrospray ionization (ESI), makes mass spectrometric studies of protein-ligand interactions possible by producing multiply charged ions of complexes and, at least

Correspondence to: D. J. Douglas; e-mail: douglas@chem.ubc.ca in some cases, preserving the tertiary and quaternary structures of noncovalent complexes in the gas phase. Electrospray-MS has relatively little sample consumption $\left(10^{-12}\right.$ to $\left.10^{-15} \mathrm{~mol}\right)$ [1] compared with techniques such as NMR or X-ray diffraction, and has therefore complemented these methods to give insight into structures of proteinprotein or protein-small-molecule complexes [2].

Attempts to elucidate fundamental biological properties or processes based on MS analysis of complexes raise a long-standing question: How closely are gas-phase and solution-phase structures and binding related? Destabilization of proteins may occur in the absence of water if water is 
preferentially involved in noncovalent interactions [3]. In some cases, protein-ligand complexes partially dissociate in the gas phase [4-6] and in other cases, nonspecific adducts or clusters, which are artefacts of mass spectrometry and do not represent solution species, can be formed $[5,7,8]$. Thus, the binding in gas-phase noncovalent complexes will not necessarily parallel solution binding. Nevertheless, some previous studies have shown that gaseous protein ions preserve, at least partially, solution-phase binding characteristics [8-14]. If conditions can be found where gas-phase binding is related to solution-phase binding, mass spectrometry may be more widely applied to systematically determine the relative binding strengths of a variety of molecules with a target protein, with the advantages over other methods of speed, sensitivity, and specificity.

In some cases, changes to conformations of an entire protein or domains of a protein, when binding a ligand, are involved in the biological function of a protein. In this study, we have used collision cross section measurements that provide information on an ion's "size" averaged over all orientations [6,10,14], and solution- and gas-phase hydrogen/deuterium exchange (H/Dx) coupled with MS, to probe the conformations of a protein and the protein binding smallmolecule inhibitors. Tandem mass spectrometry (MS/MS) has also been used to study the relative gas-phase binding strengths of ligand-bound protein ions [10, 14, 15]. [Absolute binding energies of gas-phase complexes can be measured with blackbody infrared radiative dissociation (BIRD) experiments (see for example [5, 8, 11-13])].

In solution, $\mathrm{H} / \mathrm{Dx}$ with $\mathrm{D}_{2} \mathrm{O}$, followed by $\mathrm{MS}$ analysis has been extensively employed to relate isotopic exchange rates of amide hydrogens and labile hydrogens on side chains to protein higher-level structures [16]. Hydrogens exposed on a protein's surface can be rapidly exchanged, while protected hydrogens, such as those involved in hydrogen bonding or buried inside the protein core, have much lower rates of exchange. Thus, for a given $\mathrm{pH}$ and temperature [17], higher exchange levels of a protein in solution $\mathrm{H} / \mathrm{Dx}$ indicate a more unfolded or more flexible conformation.

In comparison to solution-phase $\mathrm{H} / \mathrm{Dx}$, gas-phase $\mathrm{H} / \mathrm{Dx}$ is less well established and more difficult to interpret [18-21]. For exchange, gas-phase ions can be trapped in a variety of reagents, such as $\mathrm{D}_{2} \mathrm{O}[19,22], \mathrm{CD}_{3} \mathrm{OD}$ [21], or $\mathrm{ND}_{3}$ [18], at a pressure of $10^{-1}$ Torr in an ion mobility drift tube [23], $10^{-3}$ Torr in a quadrupole ion trap [24], or $10^{-7}$ Torr in an ICR cell $[18,22,25]$. For a reagent with a low proton affinity, such as $\mathrm{D}_{2} \mathrm{O}$, a relay mechanism has proposed for the exchange reaction $[19,20,25]$. Because of a high energy barrier $\left(E_{a}>10 \mathrm{kcal} / \mathrm{mol}\right)$ for direct reaction, $\mathrm{D}_{2} \mathrm{O}$ cannot exchange with a neutral amide and must attack at a charged site such as a lysine side chain or the N-terminus. The $\mathrm{D}_{2} \mathrm{O}$ forms a hydrogen bond to the charged site, and then can exchange with a nearby amide hydrogen. In unfolded conformations, an exchangeable site and charged site may be too widely separated to allow exchange, leading to lower exchange levels than the same protein in a more folded conformation. In this mechanism, the exchange rate is determined by the chemistry of the individual protein. For example, Evans et al. [21] found that +15 cytochrome $c$ ions confined in a 3D trap with $\mathrm{CD}_{3} \mathrm{OD}$ vapor exchange more hydrogens than +8 ions, while +10 ubiquitin ions exchange fewer hydrogens than +7 ions. Collision cross section measurements show that with both proteins the more highly charged ions generally have more unfolded conformations $[23,26]$. Freitas et al., using FTICR-MS, also found that +7 and +9 ubiquitin ions exchange hydrogens more rapidly with $\mathrm{D}_{2} \mathrm{O}$ than +8 or +10 ions [22]. In a study combining mobility measurements of cross sections with H/Dx, Valentine and Clemmer [23] found that with $+8,+9$, and +10 cytochrome $c$ ions, compact conformations exchanged fewer hydrogens than more diffuse ions of the same charge with larger cross sections, but the more highly charged ions +11 to +18 , which have substantially greater cross sections, all exchanged about the same number of hydrogens as the diffuse ions in the lower charge states. Thus, gas-phase $\mathrm{H} / \mathrm{Dx}$ does not necessarily reveal the folding state of an ion. However different H/Dx levels can reveal different conformations of a given ion.

Here, binding of the catalytic domain of the enzyme exo$1,4-\beta$-D-glycanase (cellulase xylanase or "Cex") with azasugar inhibitors, serves as a model to study conformations in solution and in the gas phase of protein-small-molecule noncovalent complexes. Cex (from the micro-organism Cellulomonas fimi) is a family 10 glycosal hydrolase. The catalytic domain of Cex folds as a $(\alpha / \beta)_{8}$-barrel, and catalyses hydrolytic cleavage of $\beta-1,4$ linked polymers of D-xylose [27]. A series of small-molecule inhibitors that noncovalently bind at the active site of the catalytic domain of Cex (Cex-cd) with high affinity have been described [27]: imidazole, lactam oxime, deoxynojirimycin $\left(\mathrm{X}_{2} \mathrm{DNJ}\right)$, isofagomine lactam $\left(\mathrm{X}_{2} \mathrm{IL}\right)$, and isofagomine $\left(\mathrm{X}_{2} \mathrm{IF}\right)$, three of which are used in this work and are shown in Figure 1. These xylobiose-derived nitrogen-containing inhibitors consist of two sugars, a common distal xylose that binds to the -2 sub-site of Cex and a distinct proximal aza-sugar that binds to the -1 sub-site $[27,28]$. Cex binds to inhibitors mainly through hydrogen bonding but also through van der Waals forces and electrostatic interactions [29]. Changing the inhibitors affects the binding of Cex to the proximal sugar, while the binding to the distal xylose is largely preserved [28]. Comparison of the interactive maps via Xray crystallographic studies with different inhibitors has been described in detail [28]. The dissociation or inhibition constants, indicating binding strengths in solution, $K_{i}$, have been determined through kinetic analysis [30], and are listed in Table 1. In solution, the Cex- $\mathrm{X}_{2} \mathrm{DNJ}$ complex has the lowest binding strength, Cex- $\mathrm{X}_{2} \mathrm{IL}$ is intermediate, and Cex $-\mathrm{X}_{2} \mathrm{IF}$ has the highest binding strength.

Previously in this lab, the binding in ions of the gas-phase complexes of wild type Cex and three variants of Cex with the noncovalent inhibitors, $\mathrm{X}_{2} \mathrm{IF}, \mathrm{X}_{2} \mathrm{IL}$, and $\mathrm{X}_{2} \mathrm{DNJ}$ was studied with collision cross section measurements and 


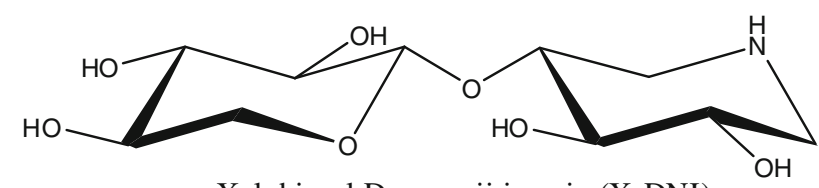

Xylobiosyl Deoxynojirimycin $\left(\mathrm{X}_{2} \mathrm{DNJ}\right)$ $\mathrm{MW}=265 \mathrm{Da}$
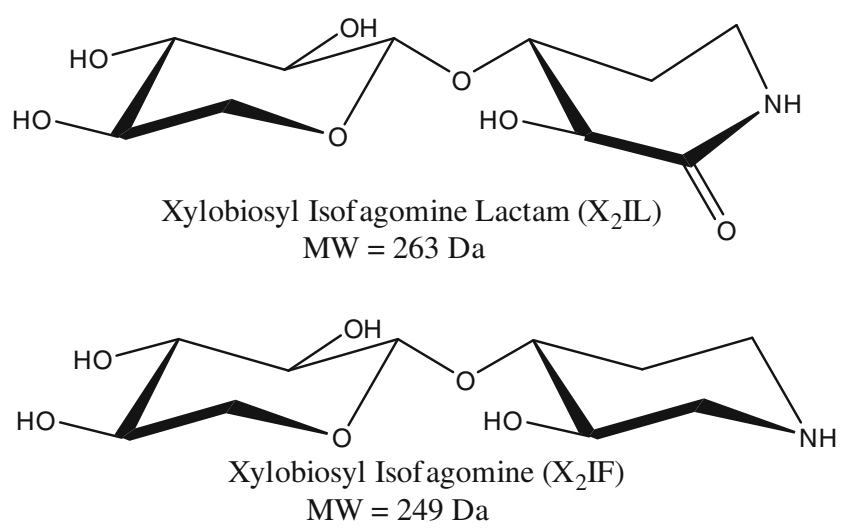

Figure 1. Structures and molecular weights (MW) of the three inhibitors. The distal xylose is on the left and the proximal aza-sugar is on the right

MS/MS experiments [14]. Mass spectra showed ions of Cex, Cex binding a single inhibitor, and a minor peak corresponding Cex binding two inhibitors. Because Cex has only a single binding site, the peak corresponding to Cex with two inhibitors was attributed to nonspecific binding. Triple quadrupole tandem MS showed that the relative binding strengths of $+9,+10$, and +11 ions of all forms of Cex binding each of the three inhibitors in the gas phase parallel the free energy changes, $\Delta G^{0}$, for binding in solution, calculated from the inhibition constants $K_{i}$ (Table 1). Complexes have lower cross sections than free protein ions, and the cross sections of the complexes decrease as the gasphase binding strengths increase. It was concluded that with Cex and these three inhibitors, all the hydrogen bonds between the inhibitors and the enzyme that are involved in solution binding are preserved in the gas-phase complexes and, therefore, that the gas-phase enzyme retains its specific solution binding characteristics.

In this study, we have extended our earlier work by using solution and gas-phase $\mathrm{H} / \mathrm{Dx}$ of Cex with three noncovalent inhibitors, $\mathrm{X}_{2} \mathrm{IF}, \mathrm{X}_{2} \mathrm{IL}$, and $\mathrm{X}_{2} \mathrm{DNJ}$, to gain further insights into the properties of the solution and gas-phase complexes.

Table 1. Inhibition Constants, Calculated Cex and Complex Concentrations, and Ratios of Complex to Cex Concentration with Initial Total Solution Concentrations of Cex and Inhibitor of $10 \mu \mathrm{M}$ and $33 \mu \mathrm{M}$ Respectively

\begin{tabular}{lcccc}
\hline Inhibitor & $K_{i}(\mu \mathrm{M})^{\mathrm{a}}$ & {$[\mathrm{Cex}](\mu \mathrm{M})$} & {$[$ Complex] $(\mu \mathrm{M})$} & {$[$ Complex $] /[$ Cex $]$} \\
\hline $\mathrm{X}_{2} \mathrm{DNJ}$ & 5.8 & 1.89 & 8.11 & 4.29 \\
$\mathrm{X}_{2} \mathrm{IL}$ & 0.33 & 0.14 & 9.86 & 70.4 \\
$\mathrm{X}_{2} \mathrm{IF}$ & 0.13 & 0.056 & 9.94 & 177 \\
\hline
\end{tabular}

${ }^{\mathrm{a}} K_{i}=[$ Cex $][$ inhibitor $] /[$ complex $]$.
Here a different version of the catalytic domain of Cex with a 6-histidine tag $\left(\mathrm{H}_{6}\right.$-Cex-cd) is used. Compared with the Cex-cd used previously (MW 34850.1 Da), $\mathrm{H}_{6}$-Cex-cd (MW $35824.9 \mathrm{Da}$ ) has three additional amino acid residues and differs in 50 residues. Despite the sequence difference, the -1 and -2 binding sub-sites are identical in these two proteins, as shown in Scheme 1. Because this enzyme has a different sequence and gives slightly different charge states in mass spectra ( +11 and +12 here, +10 and +11 in [14]), we have repeated the cross section and tandem mass spectrometry measurements with ions of +11 and +12 charge states. With the +11 ions, the results of both experiments are in good agreement with the previous study [14], indicating the sequence changes at non-binding sites do not significantly influence the binding strength or conformation of the gasphase Cex-inhibitor complexes.

Mass spectra show higher abundances of free protein than the abundances calculated to be present in solution from $K_{i}$ values. We show that these protein ions are mostly formed by dissociation of ions of the complexes in the ion sampling process. In solution, complexes have lower H/Dx levels than free Cex. The complexes and Cex from a solution with the inhibitors show the same H/Dx levels, showing that the protein is almost entirely bound in solution, as calculated, and providing additional evidence that the protein ions observed in the mass spectra are formed in the ion sampling process. As seen previously [14], cross section measurements here show ions of the complexes have more compact conformations than ions of Cex from a solution of $\mathrm{Cex}$ alone. Surprisingly, Cex ions from solutions with an inhibitor have lower cross sections than Cex ions from a (a) Cex-cd

VVKPAQA

RTTPAPGHPA RGARTALRTT

LAAAAATLVV GATVVLPAQA

ATTLKEAADG AGRDFGFALD

PNRLSEAQYK AIADSEFNLV

VAENAMKWDA TEPSQNSFSF

GAGDRVASYA ADTGKELYG $\underline{H}$

TLVWHSOLPD WAKNLNGSAF

ESAMVNHVTK VADHFEGKVA

SWDVVNEAFA DGGGRRQDSA

FQQKLGNGYI ETAFRAARAA

DPTAKLCIND YNVEGINAKS

NSLYDLVKDF KARGVPLDCV

GFQSHLIVGQ VPGDFRQNLQ

RFADLGVDVR ITELLDIRMRT

PSDATKLATQ AADYKKVVQA

CMOVTRCQGV TVWGITGAS (b) H6-Cex-cd

ASHH HHHHIEGRAS

ATTLKEAADG AGRDFGFALD PNRLSEAQYK AIADSEFNLV VAENAMKWDA TEPSQNSFSF GAGDRVASYA ADTGKELYGH ESAMVNHVTK VADHFEGKVA SWDVVNEAFA DGGGRRQDSA FQQKLGNGYI ETAFRAARAA DPTAKLCIND YNVEGINAKS NSLYDLVKDF KARGVPLDCV GFQSHLIVGQ VPGDFRQNLQ RFADLGVDVR ITELDIRMRT PSDATKLATQ AADYKKVVQA CMOVTRCQGV TVWGITDKYS WVPDVFPGEG AALVWDASYA TLVWHSOLPD WAKNLNGSAF KKPAYAAVME AFGAS

\section{$X$ : binding sites for the distal xylose from $\mathrm{X}_{2} \mathrm{DNJ}$}

$\underline{\mathrm{X}}$ : binding sites for the proximal sugar from $\mathrm{X}_{2} \mathrm{DNJ}$

Scheme 1. Sequence of the Cex catalytic domain, (a) Cex-cd used in [14] and (b) $\mathrm{H}_{6}$-Cex-cd used in this work. Residues involved in binding the proximal and distal xyloses of $\mathrm{X}_{2} \mathrm{DNJ}$ are shown in red. Differences in sequence at the $\mathrm{N}$ and $\mathrm{C}$ termini are shown in blue 
solution of Cex alone, and cross sections similar to the complexes, suggesting that these Cex ions "remember" their solution conformations. In gas-phase $\mathrm{H} / \mathrm{Dx}$, ions of the complexes generally show greater $\mathrm{H} / \mathrm{Dx}$ levels than ions of Cex with the same charge. Unlike the cross sections, the $\mathrm{H} / \mathrm{Dx}$ levels of the complexes do not correlate with the relative gas-phase binding strengths measured by MS/MS, suggesting the ions may fold or unfold when trapped for up to $15 \mathrm{~s}$ for H/Dx. Cex ions from solutions with Cex alone, and Cex ions from solutions with an inhibitor, which have different cross sections, show the same H/Dx levels after $15 \mathrm{~s}$, also suggesting ions may fold or unfold when trapped for H/Dx. Overall, the gas-phase complexes have more compact conformations than free protein ions on the ca. $1 \mathrm{~ms}$ time scale of cross section measurements, and retain different and possibly more folded conformations than the Cex ions on the seconds time scale of $\mathrm{H} / \mathrm{Dx}$ experiments.

\section{Experimental}

\section{Triple Quadrupole MS System and Solution H/Dx}

For solution H/Dx experiments, a home-built triple quadrupole mass spectrometer described previously was used [14, 31]. Stock solutions of Cex and inhibitors were mixed to a concentration of $160 \mu \mathrm{M}$ of Cex and $528 \mu \mathrm{M}$ of inhibitor. For $\mathrm{H} / \mathrm{Dx}$ analysis, the solutions were diluted in $10 \mathrm{mM}$ deuterated ammonium acetate and 90/10 (vol/vol) $\mathrm{D}_{2} \mathrm{O} /$ $\mathrm{CD}_{3} \mathrm{OD}$, to a final concentration of $10 \mu \mathrm{M}$ in Cex and $33 \mu \mathrm{M}$ in inhibitor. The same solvent was used for H/Dx of Cex alone. The corrected $\mathrm{pD}$ in solution was 7.1 at room temperature $(\mathrm{pD}=\mathrm{pH}$ meter reading +0.4$)$ [32]. Samples in $\mathrm{D}_{2} \mathrm{O}$ were immediately infused into a pneumatically assisted ESI source with a syringe pump (Harvard Apparatus, St. Laurent, PQ, Canada) at $1 \mu \mathrm{L} / \mathrm{min}$. Positively charged gaseous ions pass through an aperture in a curtain plate, an orifice-skimmer region with a voltage difference $\left(\Delta V_{\text {ori-ski }}\right)$ of $100 \mathrm{~V}$, then enter a quadrupole ion guide Q0 operated at 9 mTorr $\left(\mathrm{N}_{2}\right)$ for collision cooling [33]. After passing through a short radio frequency quadrupole, ions enter a quadrupole Q1 for mass analysis with quadrupoles Q2 and Q3 used as ion guides. Pulse counting is used for ion detection. Spectra were then recorded repeatedly at different time intervals for one hour. The number of hydrogens exchanged is calculated from the mass difference between the intact protein and the deuterated protein. ES Tuning mix (Agilent, Santa Clara CA, USA) was used for mass calibration.

\section{Collision Cross Sections and MS/MS}

For cross section and MS/MS measurements, experiments were done with the same triple quadrupole mass spectrometer with modifications to the ion sampling and vacuum system to increase sensitivity, as described in [6]. For cross section experiments, Q1 is operated as an ion guide and Q3 as a mass filter. For MS/MS, both Q1 and Q3 are operated as mass filters and Q2 as a collision cell.

When ions pass through low-density gas (argon) in Q2, they lose axial kinetic energy through multiple non-reactive collisions. The energy losses can be related to collision cross sections with an aerodynamic drag model [34] by [6, $10,31,35]$,

$$
\frac{E}{E^{0}}=\exp \left(\frac{-C_{d} n m_{2} l \sigma}{m_{1}}\right)
$$

where $E$ is the ion kinetic energy at the cell exit, $E^{0}$ is the ion kinetic energy at the cell entrance, $C_{d}$ is a drag coefficient, $n$ is the gas number density, $m_{1}$ is the protein ion mass, $m_{2}$ is the collision gas mass, $l$ is the length of the collision cell and $\sigma$ is the collision cross section. To determine energy losses, the Q2 pressure was systematically increased from 0.0 to 1.2 mTorr, measured with a precision capacitance manometer (model 120AA; MKS Instruments, Boulder, CO, USA). Stopping curves were measured at each pressure by systematically increasing the Q3 rod offset voltage. Cross sections were then calculated by plotting $-\ln \frac{E}{E_{0}}$ versus $\frac{C_{d} n m_{2} l}{m_{1}}$.

In MS/MS, precursor complex ions are mass selected in Q1, and have multiple energetic collisions with $\mathrm{Ar}$ in Q2 leading to dissociation. Fragment ions are then mass analyzed in Q3. To compare relative binding strengths of the complexes, the total internal energies added to ions in Q2, $\Delta E_{\text {int }}$, are determined with a collision model [10, 14, 36], which corrects for losses of kinetic energy as the precursor ions move through the cell and for the different numbers of collisions of ions with different collision cross sections. Values of $\Delta E_{\text {int }}$ are calculated from

$$
\Delta E_{\mathrm{int}}=\Phi \frac{m_{2}}{M} E^{0} \frac{m_{1}}{m_{2}} \frac{1}{C_{d}}\left[1-\exp \left(-\frac{C_{d} n m_{2} \sigma l}{m_{1}}\right)\right]
$$

where $\Phi$ is the average fraction of center-of-mass kinetic energy transferred to internal energy in a collision (taken to be 1.0), and $M=m_{1}+m_{2}$. The initial kinetic energy of the complex ions per charge, $E^{0}$, is taken as the difference in rod offsets between Q0 and Q2 which causes one half of the precursor ions to dissociate in Q2. For each complex, values of $\Delta E_{\text {int }}$ were determined with at least three cell pressures from 0.5 to 2.74 mtorr. At each pressure, the time available for reaction occurring over a length of ca. $5 \mathrm{~cm}$ near the cell exit was determined as previously described $[14,36]$.

\section{Trap-TOF MS System and Gas-Phase H/Dx}

Gas-phase H/Dx experiments were done with a home-built linear quadrupole ion trap reflectron time-of-flight mass spectrometer system (LIT-TOF MS) [24, 35, 37, 38]. Ions, generated by pneumatically assisted ESI, pass through a curtain plate, a dry nitrogen curtain gas, and an orificeskimmer region with a voltage difference of 150 to $180 \mathrm{~V}$. In control experiments, Cex or Cex complex ions continuously pass through a chamber with two consecutive ion guide 
quadrupoles, Q0 $\left(\Delta V_{s k i-Q 0}=5 \mathrm{~V}\right)$ and $\mathrm{Q} 1$, in the presence of $10 \mathrm{mTorr}$ of $\mathrm{N}_{2}$, with the pressure controlled by a gate valve between the chamber and a turbo pump. For H/Dx experiments, the chamber was maintained at $4 \mathrm{mTorr}$ of $\mathrm{N}_{2}$, then filled with 6 mTorr of $\mathrm{D}_{2} \mathrm{O}$ vapor controlled with a needle valve (SS-SS4-Al; Swagelok, Solon, OH, USA), to a total pressure of 10 mTorr. Ions are confined axially in Q1 by adding timed DC stopping potentials to the entrance and exit lenses for up to $15 \mathrm{~s}$ [10]. Following this, the exit lens voltage is lowered and ions drain through a stack of lens for mass analysis with a reflectron TOF. The extent of exchange is determined by the mass increase between the control and exchange experiments. Mass spectra for non-deuterated $\mathrm{Cex}$ were recorded repeatedly at the beginning and the end of each trial to ensure no instrumental mass drift occurred. A solution of CsI was used for mass calibration.

\section{Solutions and Reagents}

Cex and the inhibitors were provided by Stephen Withers' group, UBC. Cex stock solution $(8 \mu \mathrm{L}, 20 \mathrm{mg} / \mathrm{mL})$ was desalted by buffer exchange with $550 \mu \mathrm{L} \mathrm{NH} \mathrm{N}_{4} \mathrm{Ac}(12.5 \mathrm{mM})$ with a $10 \mathrm{kDa}$ MWCO membrane (Millipore; Carrigtwohill Co., Cork, Ireland), with centrifugation at $11,000 \mathrm{rpm}$ for 10 min each, with eight cycles (MSE MicroCentaur; London, UK), to give a pure Cex stock solution of $200 \mu \mathrm{M}$ in $12.5 \mathrm{mM} \mathrm{NH}_{4} \mathrm{Ac}$.

For mass spectra of Cex alone, the Cex stock solution was diluted to $10 \mu \mathrm{M}$ in $10 \mathrm{mM} \mathrm{NH}_{4} \mathrm{Ac}$ and $\mathrm{H}_{2} \mathrm{O} / \mathrm{MeOH} 90 /$ 10 (vol/vol) at pH 6.6 measured with a Accumet model 15

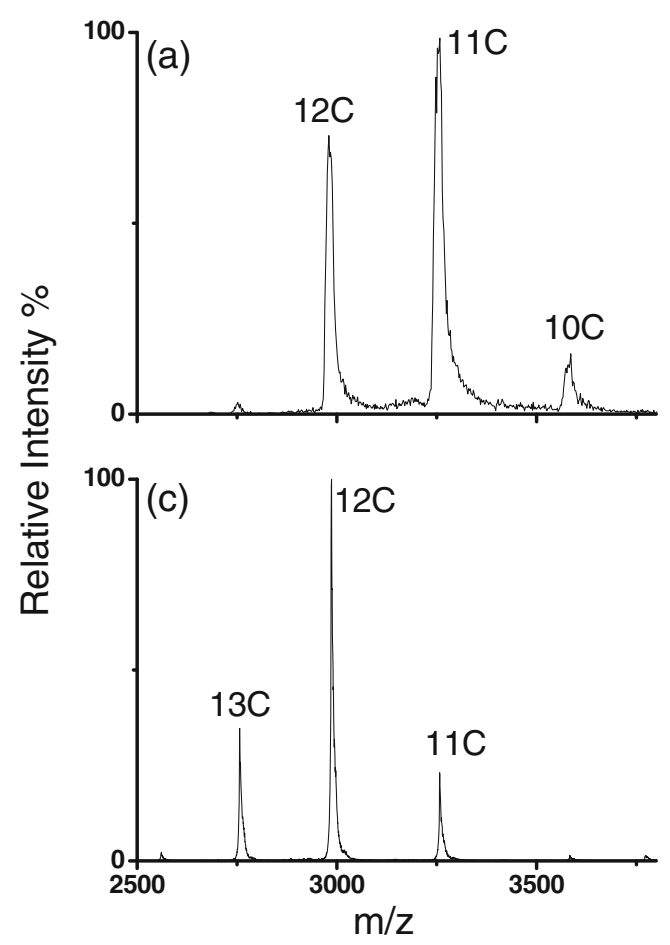

pH meter (Fisher Scientific, Fairlawn, NJ, USA). For mass spectra of the complexes, the inhibitors were mixed with Cex at room temperature. The final solutions contained $10 \mu \mathrm{M}$ Cex and $33 \mu \mathrm{M}$ inhibitor with the same solvent composition as the solution with Cex alone. Deuterated solutions are described above.

Cesium iodide (99.99\%) was from Sigma-Aldrich, St. Louis, MO, USA. Methanol (HPLC grade) and $\mathrm{NH}_{4} \mathrm{Ac}$ (ACS grade) were from Fisher Scientific, Fairlawn, NJ, USA. Deuterium oxide (99.9\%) and methanol-d4 (99.8\%) were from Cambridge Isotope Laboratories, Inc., Andover, MA, USA. Nitrogen and argon (99.999\% manufacturer's stated purity) were from Praxair, Mississauga, ON, Canada.

\section{Results and Discussion}

\section{Mass Spectra}

Figure $2 \mathrm{a}$ and $\mathrm{b}$ show ESI triple quadrupole MS spectra of Cex and $\mathrm{Cex}-\mathrm{X}_{2} \mathrm{IL}$ complexes over the +10 to +14 charge state region. Cex binds specifically to one inhibitor molecule. With both $C e x$ and the complexes, the +11 and +12 ions dominate the mass spectra, with +10 ions of lower intensity. With the two other complexes, Cex- $\mathrm{X}_{2} \mathrm{DNJ}$ and $\mathrm{Cex}-\mathrm{X}_{2} \mathrm{IF}$, spectra were similar with slightly less intense +12 ions and slightly more intense +10 ions (data not shown). Compared with previous spectra with Cex-cd with the triple quadrupole MS [14], the $\mathrm{H}_{6^{-}}$ Cex-cd protein and its complexes produce significantly more abundant +12 ions and less abundant +10 ions, and no +9 ions. This was seen with both our old and new ion sampling

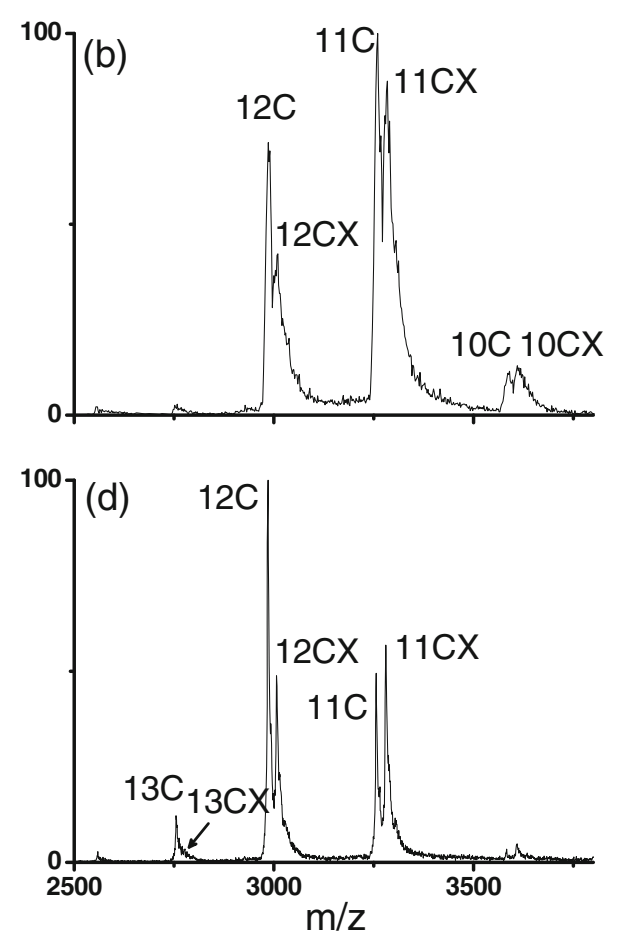

Figure 2. ESI MS spectra recorded with the triple quadrupole $M S$ of (a) Cex alone and (b) Cex complexes with $\mathrm{X}_{2} \mathrm{IL}$, and with the q-TOF MS of (c) Cex alone and (d) Cex with $\mathrm{X}_{2} \mathrm{IL}$. Charges are labeled. Notation: C, Cex; CX, Cex-inhibitor complex 
interfaces, indicating the differences in mass spectra do not come from the instrumental changes but may be attributed to the sequence differences. The six histidine tags near the $\mathrm{N}$-terminus of $\mathrm{H}_{6}$-Cex-cd may increase the charging of the protein.

Figure $2 \mathrm{c}$ and $\mathrm{d}$ show mass spectra of $\mathrm{Cex}$ and $\mathrm{Cex}-\mathrm{X}_{2} \mathrm{IL}$ complexes recorded with the q-TOF MS, which gives higher resolution and better peak shapes. Ions with +11 to +13 charges appear in the MS spectra. On average the ions appear somewhat more highly charged, compared with spectra with the triple quadrupole MS. This may due to the different ion sampling processes and instrumental operating conditions of these two systems. When Cex binds the inhibitor, the +13 ions decrease in intensity and the +11 ions increase in intensity. Similar spectra are seen with $\mathrm{Cex}-\mathrm{X}_{2} \mathrm{DNJ}$ and $\mathrm{Cex}-\mathrm{X}_{2} \mathrm{IF}$.

The ratios of intensities of complex ions to Cex ions in the mass spectra do not match the ratios of the solution abundances calculated from the values of $K_{i}$, listed in Table 1 . The observed ratios are substantially affected by the instrument operating conditions. In experiments with the triple quadrupole MS, with the orifice-skimmer voltage difference kept at $100 \mathrm{~V}$, increasing the voltage difference between the skimmer and Q0 $\left(\Delta V_{\text {ski-Q0 }}\right)$ produces more Cex ions (spectra not shown) because the increased internal energy added to the complexes in Q0 causes greater dissociation of the complexes. For example, if $\Delta V_{s k i-Q 0}$ is set to $30 \mathrm{~V}$, almost equal levels of ions of Cex and complexes are seen. If $\Delta V_{s k i-Q 0}$ is decreased to $10 \mathrm{~V}$, with $\mathrm{Cex}-\mathrm{X}_{2} \mathrm{DNJ}$, the ratio of the intensities of ions of the complex to Cex ions increases to about 4 tol, similar to the calculated abundance ratio in solution. At $\Delta V_{\text {ski-Q0 }}=10 \mathrm{~V}$, with $\mathrm{Cex}-\mathrm{X}_{2} \mathrm{IL}$ and $\mathrm{Cex}-\mathrm{X}_{2} \mathrm{IF}$ solutions, the intensity ratios of Cex ions to complex ions are less than 0.1 and 0.05 , respectively. In experiments with the q-TOF MS, where the skimmer-Q0 voltage difference is only $5.0 \mathrm{~V}$, the orificeskimmer voltage difference affects the ratio of complex to Cex ions. Higher voltage differences lead to more dissociation of the complexes. The relatively lower abundances of complexes in mass spectra compared with calculated solution abundances then derive at least in part from dissociation of complexes in the ion sampling interfaces.

\section{Collision Cross Sections}

Table 2 shows collision cross sections of Cex and Cexinhibitor complex ions with +11 and +12 charges. Uncertainties are standard deviations of three separate measure- ments. For Cex ions, cross sections were determined in two ways: first, with Cex ions from a solution containing only Cex, and second with Cex ions from solutions of Cex and the inhibitors. With all three inhibitors, Cex-inhibitor complexes (CX) have cross sections lower than Cex ions from a solution of Cex alone, as seen in [14], indicating a more compact structure is adopted by the gas-phase complex ions. Among the different complexes, Cex- $\mathrm{X}_{2} \mathrm{DNJ}$ and Cex $-\mathrm{X}_{2} \mathrm{IL}$ have similar cross sections, $11 \%$ larger than cross sections of $\mathrm{Cex}-\mathrm{X}_{2} \mathrm{IF}$. This is reasonable because Cex- $\mathrm{X}_{2} \mathrm{IF}$ also has the strongest binding in the gas phase (see below). For the +11 ions of Cex and Cex-inhibitor complexes, where comparisons are possible, the cross sections here agree with those of Tesic et al. [14] within about $2.0 \%$. Thus the global conformations of the protein and complexes are unchanged by the differences in the sequences remote from the binding site. The decrease of cross sections with increasing binding strength in the ions is purely a gas-phase effect. Structures of apo-Cex-cd and its complexes were reported to be "essentially identical" based on NMR [39] and X-ray crystallographic analysis [28, 40, 41]. For the complex ions, and Cex ions formed from the solutions containing an inhibitor, the +12 ions have somewhat larger cross sections than the +11 ions. Adding one charge is sufficient to change the conformations. However, within the uncertainties, this effect is not seen with the more unfolded ions of Cex formed from a solution of Cex alone.

Cex ions (C) from solutions of the complexes are also more compact than Cex ions from a solution of Cex alone. With $\mathrm{X}_{2} \mathrm{DNJ}$ and $\mathrm{X}_{2} \mathrm{IL}, \mathrm{Cex}$ and complex ions gave similar cross sections (within $5 \%$ ). With $\mathrm{X}_{2} \mathrm{IF}$, Cex shows a $17 \%$ larger cross section than the complex but still $7 \%$ less than Cex from a solution of Cex alone. As discussed above, these Cex ions are mostly formed when complexes are injected into and dissociate in Q0. Interestingly, these Cex ions apparently retain some memory of their origin on the ca. 1 ms time scale of the collision cross section measurements. Solution H/Dx provides additional evidence that these Cex ions are not present in solution, but are formed in the ion sampling process (see below). Knapman et al. [42] measured cross sections of fragment ions formed by loss of monomers in MS/MS experiments with several protein-protein complexes. In some cases, GroEL (a 14-mer), hemoglobin (a tetramer), and a hexamer of a virus capsid protein, MS2, the fragment ions collapsed and had substantially smaller cross sections than expected from a simple removal of a monomer

Table 2. Collision Cross Sections $\left(\AA^{2}\right)$ of Ions of Cex and Its Complexes

\begin{tabular}{|c|c|c|c|c|c|c|c|}
\hline \multirow[t]{2}{*}{ Charge } & \multirow[t]{2}{*}{ Cex } & \multicolumn{2}{|c|}{$\mathrm{Cex}-\mathrm{X}_{2} \mathrm{DNJ}$} & \multicolumn{2}{|c|}{ Cex- $\mathrm{X}_{2} \mathrm{IL}$} & \multicolumn{2}{|c|}{$\mathrm{Cex}-\mathrm{X}_{2} \mathrm{IF}$} \\
\hline & & $\mathrm{C}^{\mathrm{a}}$ & $\mathrm{CX}^{\mathrm{b}}$ & $\mathrm{C}$ & $\mathrm{CX}$ & $\mathrm{C}$ & $\mathrm{CX}$ \\
\hline+11 & $1816 \pm 57$ & $1566 \pm 67$ & $1545 \pm 53$ & $1602 \pm 59$ & $1552 \pm 14$ & $1621 \pm 52$ & $1387 \pm 48$ \\
\hline+12 & $1833 \pm 62$ & $1666 \pm 69$ & $1617 \pm 39$ & $1671 \pm 47$ & $1594 \pm 53$ & $1709 \pm 39$ & $1454 \pm 49$ \\
\hline
\end{tabular}

${ }^{\mathrm{a}} \mathrm{C}=\mathrm{Cex}$ from a mixture of $\mathrm{Cex}$ and inhibitor.

${ }^{\mathrm{b}} \mathrm{CX}=\mathrm{Cex}$-inhibitor complex. 
from the multimer. However, with major histocompatibility complex class 1 (MHC1) and a decamer of the virus capsid protein, fragment ions had cross sections close to what was expected from the species within the complex, and the fragment ions "remembered" their conformations within the complex. The dissociation of this protein-small-molecule complex cannot be compared directly with the dissociation of a protein-protein complex, but it is interesting that in both cases the proteins appear to retain their conformations within the complex after dissociation.

\section{MS/MS of Noncovalent Complexes}

In MS/MS, an enzyme-inhibitor complex ion dissociates to one Cex ion plus a neutral or charged inhibitor. Precursor ions of higher charge $(+12)$ give higher yields of charged inhibitor loss than ions with lower charges $(+10$ and +11$)$. This was not observed in the previous study with ions of +9 to +11 charges [14]. This charge-dependent MS/MS is most evident with $\mathrm{Cex}-\mathrm{X}_{2} \mathrm{DNJ}$. With +10 ions, neutral inhibitor loss dominates. With +11 ions, the ratio of neutral to charged inhibitor loss is approximately 3 to 1 , while +12 ions dissociate by losing neutral and charged inhibitor almost equally. With $\mathrm{X}_{2} \mathrm{IL}$, approximately $4 \%$ charged inhibitor loss was found with the +12 charged complexes, while the +11 and +10 ions showed mainly neutral loss. Cex $-\mathrm{X}_{2} \mathrm{IF}$ showed predominantly neutral inhibitor loss, consistent with previous results [14].

To compare gas-phase binding strengths quantitatively, the internal energy added to ions in the collision cell, $\Delta E_{\text {int }}$, was determined at different cell pressures, which have different times available for reaction. For each complex, $\Delta E_{i n t}$ was plotted vs. the time available for reaction, with the results shown in Figure 3. Only small differences in values of $\Delta E_{\text {int }}$ between the +11 and +12 ions were observed for Cex $-\mathrm{X}_{2} \mathrm{IL}$ and Cex $-\mathrm{X}_{2} \mathrm{IF}$. With $+12 \mathrm{Cex}-\mathrm{X}_{2} \mathrm{DNJ}$ ions, less internal energy, $\Delta E_{\text {int }}$, is required for dissociation than

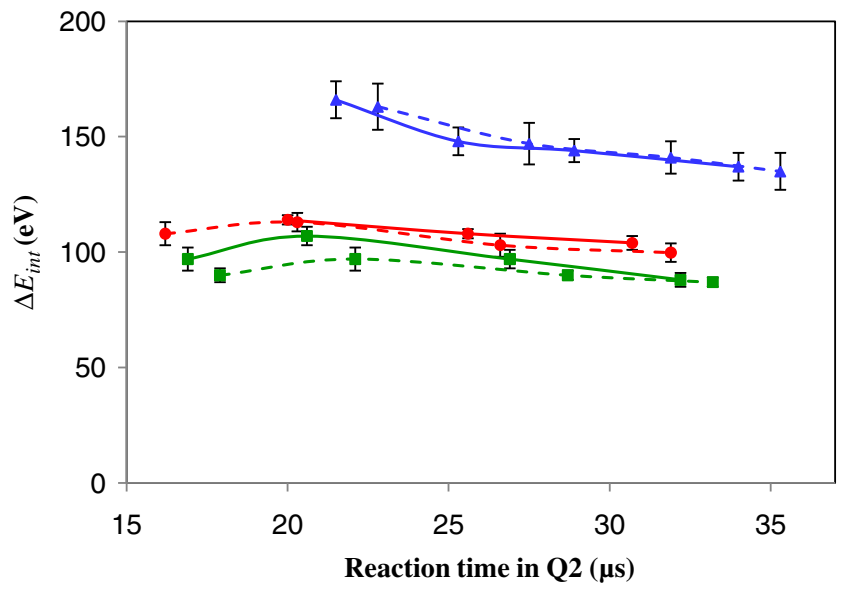

Figure 3. Values of $\Delta E_{\text {int }}$ versus the time available for reaction for the +11 (solid lines) and +12 (dashed lines) ions of Cex $-\mathrm{X}_{2} \mathrm{DNJ}$ (green), Cex- $\mathrm{X}_{2} \mathrm{IL}$ (red), and Cex- $\mathrm{X}_{2} \mathrm{IF}$ (blue)
Table 3. Internal Energies (eV) Required to Cause 50\% Dissociation of the Complexes in $25 \mu \mathrm{s}$

\begin{tabular}{lll}
\hline Ion & \multicolumn{1}{c}{+11} & +12 \\
\hline Cex-X $-\mathrm{X}_{2} \mathrm{DNJ}$ & $100.5 \pm 5$ & $94.7 \pm 5$ \\
Cex-X $\mathrm{IL}$ & $108.5 \pm 3$ & $105.5 \pm 5$ \\
Cex-X $\mathrm{IF}$ & $149 \pm 8$ & $155 \pm 10$ \\
\hline
\end{tabular}

with $+11 \mathrm{Cex}-\mathrm{X}_{2} \mathrm{DNJ}$ ions, possibly because with the charged inhibitor loss, Columbic repulsion lowers the additional internal energy required [15]. The order of the binding strengths in the gas-phase ions is: $\mathrm{X}_{2} \mathrm{IF}>\mathrm{X}_{2} \mathrm{IL}>$ $\mathrm{X}_{2} \mathrm{DNJ}$, the same order as the values of $\Delta G^{0}$ for dissociation in solution, as was found in [14]. For direct comparison with the previous results [14], the values of $\Delta E_{\text {int }}$ required to induce dissociation in $25 \mu$ s were read from the graph, and are shown in Table 3 . With the +11 ions, where comparisons are possible, the $\Delta E_{\text {int }}$ values agree with those of Tesic et al. [14] within $7 \%$ or better, and within the combined uncertainties, indicating the gas-phase noncovalent binding energy of this version of Cex is not significantly influenced by the sequence alteration.

\section{Solution $H / D x$}

Measurements of solution H/D exchange were performed with solutions containing Cex only, then solutions of Cex with an inhibitor. The exchange levels were monitored at different time intervals over $1 \mathrm{~h}$, with an example shown in Figure 4 for +11 Cex $-\mathrm{X}_{2} \mathrm{IF}$ ions. The first data were collected 3 min after the exchange started and at this time the exchange was already nearly complete. At $\mathrm{pD} 7.1,99 \%$ of the unprotected backbone amide and side-chain hydrogens are expected to exchange within $1 \mathrm{~s}$ [17, 43]. During the exchange, single peaks that moved to higher mass were seen, indicating an EX2 mechanism [16], as expected [39]. The numbers of hydrogens exchanged after $60 \mathrm{~min}$ by Cex

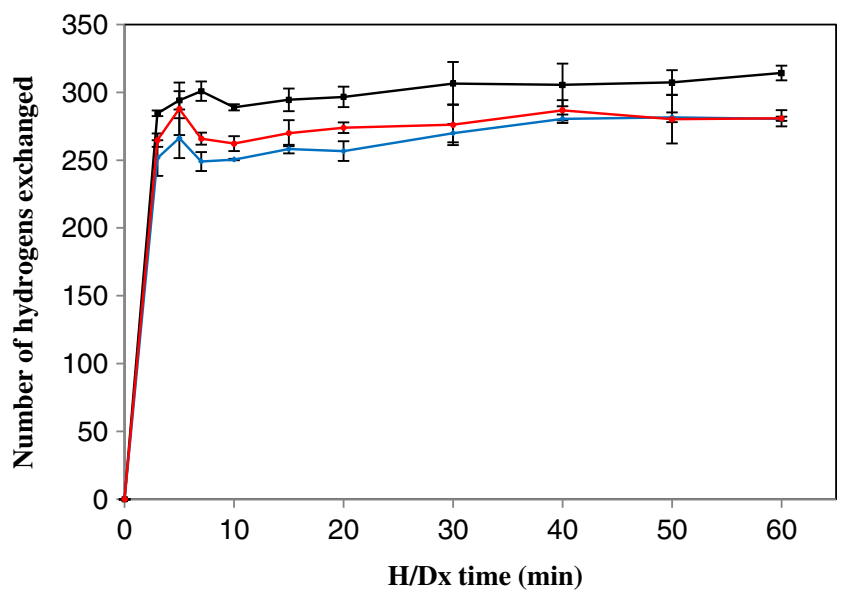

Figure 4. Numbers of hydrogens exchanged in solution as a function of reaction time measured with +11 Cex ions from a solution of Cex alone (black), from a solution of Cex with $\mathrm{X}_{2} \mathrm{IF}$ (blue), and with +11 ions of the Cex- $\mathrm{X}_{2} \mathrm{IF}$ complex (red) 
and the complexes, measured with the +11 ions, are listed in Table 4. The same exchange levels were measured on the +10 and +12 ions (data not shown). As shown in Table 4, Cex ions from a solution of Cex alone consistently show higher exchange levels than Cex-inhibitor complexes and Cex ions from a solution containing the inhibitors. No differences in $\mathrm{H} / \mathrm{Dx}$ levels were seen with Cex binding to different inhibitors. These noncovalent complexes all have basically the same X-ray structures [28, 39-41]. Apparently the different inhibitors stabilize the protein against fluctuations that lead to $\mathrm{H} / \mathrm{D}$ exchange to the same extent.

The H/Dx levels of Cex ions from solutions containing the inhibitors were also measured. Cex from solutions containing the inhibitors show the same exchange levels as the complexes (Figure 4 and Table 4). As discussed above, these Cex ions are likely formed from dissociation of ions in the skimmer-Q0 region of the triple quadrupole. Because the deuterium uptake is complete in solution, and, from the values of $K_{i}$, it is calculated that Cex is almost entirely bound to inhibitors in solution (Table 1), these Cex ions come from Cex that is bound to inhibitors in solution and show the same exchange levels as the complexes.

As shown in Table 4, after one hour H/D exchange, Cex from a solution of Cex alone exchanges $314 \pm 5$ hydrogens, $38 \pm 11(14 \%)$ more than Cex $-\mathrm{X}_{2} \mathrm{DNJ}$ and $33 \pm 11(12 \%)$ more than $\mathrm{Cex}-\mathrm{X}_{2} \mathrm{IL}$ and $\mathrm{Cex}-\mathrm{X}_{2} \mathrm{IF}$. Two factors account for the lower H/Dx levels with complexes. First, the interactive maps [28] show that about 10 hydrogens are involved in intermolecular hydrogen bonding between the inhibitor and enzyme. Second, NMR studies by Poon et al. $[39,44]$, show the enzyme has less flexible conformations after binding the inhibitors, thus reducing fluctuations around binding sites. In their studies, binding of the inhibitor 2,4-dinitrophenyl 2-deoxy-2-fluro- $\beta$-cellobioside to Cex-cd was described. This inhibitor has the same noncovalent binding through hydrogen bonds to the enzyme as the inhibitors used here, except that it forms a covalent bond to Glu 233 with loss of dinitrophenol. Binding to the inhibitor clearly stabilized fluctuations of Cex-cd, leading to reduced hydrogen exchange in both the short-term $(<65 \mathrm{~h})$ and the long-term $(500 \mathrm{~d})$ of at least 12 amide protons and their side chains that are clustered around the active site. From their results, it can be calculated that at least 22 hydrogens are protected when the inhibitor is bound. This is fewer than we find because in the NMR experiment not all residues were

Table 4. Numbers of Hydrogens Exchanged After $1 \mathrm{~h}$ of Solution H/Dx

\begin{tabular}{lll}
\hline Cex alone & & $314 \pm 5$ \\
\hline Cex-X ${ }_{2}$ DNJ & C $^{\mathrm{a}}$ & $281 \pm 14$ \\
Cex-X $\mathrm{X}$ IL & CX & $276 \pm 10$ \\
& $\mathrm{C}$ & $280 \pm 6$ \\
Cex $-\mathrm{X}_{2} \mathrm{IF}$ & $\mathrm{CX}$ & $281 \pm 10$ \\
& $\mathrm{C}$ & $280 \pm 2$ \\
& $\mathrm{CX}$ & $281 \pm 6$ \\
\hline
\end{tabular}

${ }^{\mathrm{a}} \mathrm{C}=\mathrm{Cex}$ from a solution of Cex-inhibitor mixture.

${ }^{\mathrm{b}} \mathrm{CX}=\mathrm{Cex}$-inhibitor complex. resolved and, thus, only a lower limit to the number of protected hydrogens was given. Because the protein in the complex has the same global conformation as the free protein in solution [28, 39-41], it is the loss of fluctuations that open up the protein for $\mathrm{H} / \mathrm{Dx}$ that reduces the number of hydrogens exchanged.

\section{Gas-Phase H/Dx}

Gas-phase H/Dx experiments were performed with the +11 , +12 , and +13 ions of Cex and Cex-inhibitor complexes with the q-TOF MS. During the exchange, no bimodal isotopic distributions were seen, suggesting that a single conformation is present for each of the ions, or that the ions fluctuate between different conformations on a time scale much shorter than the exchange time (EX2 mechanism). As an example, the numbers of hydrogens exchanged versus trapping time by gas-phase ions of Cex and gas-phase ions of Cex $-\mathrm{X}_{2} \mathrm{DNJ}$ complexes are shown in Figure 5. In the first $2 \mathrm{~s}$, Cex and the complexes exchange about the same number of hydrogens. Following this, ions of the complexes show somewhat greater rates of exchange than ions of Cex. After ca. $12 \mathrm{~s}$, the exchange is mostly complete. Similar exchange behavior is seen with the Cex- $\mathrm{X}_{2} \mathrm{IF}$ and Cex- $\mathrm{X}_{2} \mathrm{IL}$ ions (data not shown). The numbers of hydrogens exchanged after $15 \mathrm{~s}$ of trapping in $\mathrm{D}_{2} \mathrm{O}$ vapor are shown in Table $5 \mathrm{a}$. For a given charge, the complexes Cex- $\mathrm{X}_{2} \mathrm{DNJ}$ and $\mathrm{Cex}-$ $\mathrm{X}_{2} \mathrm{IF}$ exchange about the same number of hydrogens. The +11 and +12 ions of Cex $-\mathrm{X}_{2} \mathrm{IL}$ exchange fewer hydrogens than the +11 and +12 ions of $C e x-X_{2}$ DNJ and Cex $-X_{2} I F$, but $+13 \mathrm{Cex}-\mathrm{X}_{2} \mathrm{IL}$ ions exchange almost the same number as +13 ions of Cex $-\mathrm{X}_{2} \mathrm{DNJ}$ and $\mathrm{Cex}-\mathrm{X}_{2} \mathrm{IF}$. The binding strengths in the gas phase follow the order: $\mathrm{Cex}-\mathrm{X}_{2} \mathrm{IF}>\mathrm{Cex}-$ $\mathrm{X}_{2} \mathrm{IL}>\mathrm{Cex}-\mathrm{X}_{2} \mathrm{DNJ}$ (Table 3). Thus, the numbers of hydrogens exchanged do not correlate with the binding energies.

Unexpectedly, the +11 and +12 ions of Cex, exchange fewer hydrogens than the +11 and +12 ions of the complexes with all three inhibitors (Table 5a). The differences in exchange levels between Cex and the complexes decrease with increasing charge state. The $+13 \mathrm{Cex}$ ions and the +13 ions of the complexes exchange about the same number of hydrogens. Adding charges to the complex ions slightly increases the collision cross sections (Table 2), but apparently decreases the number of hydrogens exchanged (Table 5a). Exchange levels of +12 and +13 ions relative to +11 ions are shown in Table 5 b. The decreases in relative exchange levels with increasing charge are about the same for ions of the protein alone and the three complexes.

While Cex ions from a solution of protein alone show greater cross sections than Cex ions from a solution containing the inhibitors, Cex ions formed from these two solutions exchange the same number of hydrogens after $15 \mathrm{~s}$ of $\mathrm{H} / \mathrm{Dx}$ in the gas phase. If Cex ions formed from dissociation of complex ions in the orifice-skimmer region of the q-TOF also have more compact conformations than Cex ions from a solution of Cex alone, they appear to adopt 

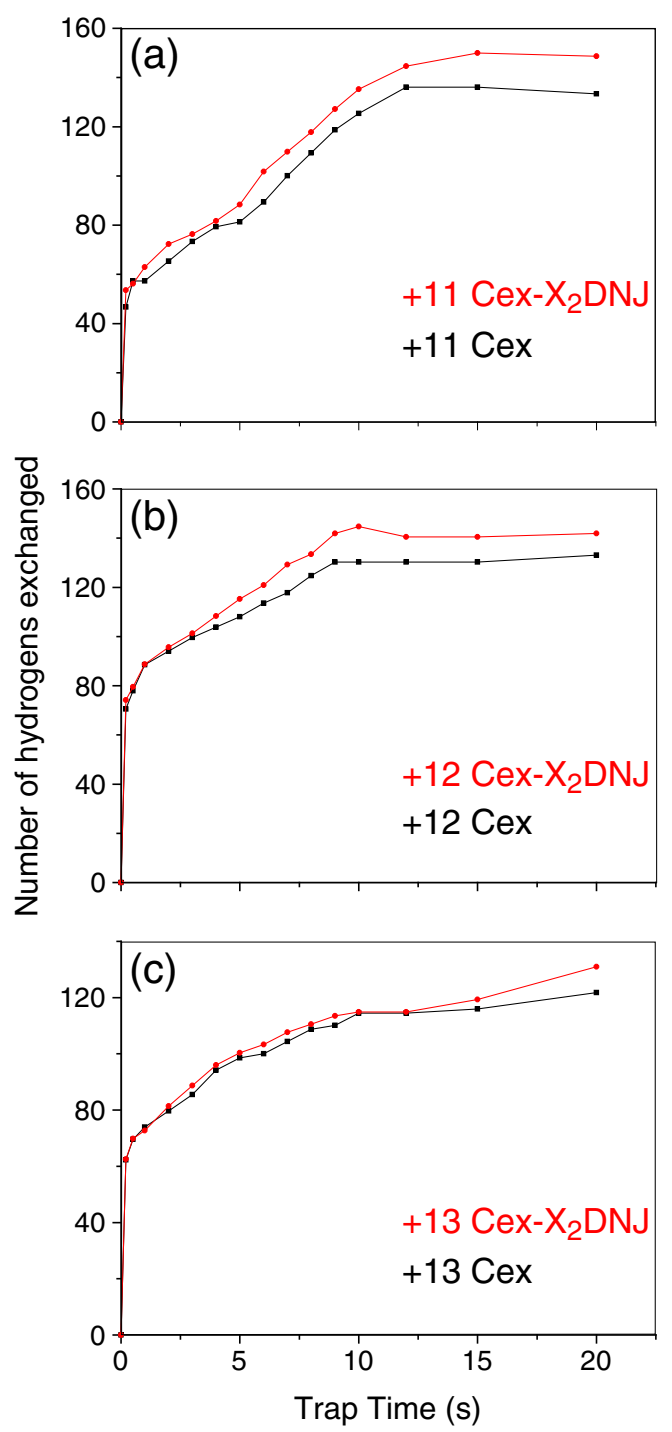

Figure 5. Gas-phase H/Dx levels as a function of trap time of Cex (black) and Cex- $\mathrm{X}_{2} \mathrm{DNJ}$ (red) ions with (a) +11 , (b) +12 , and (c) +13 charges

similar conformations when trapped in the gas phase for up to $15 \mathrm{~s}$.

Gas-phase H/D exchange occurs via different mechanisms than solution H/Dx, as discussed above. Sites that can readily exchange in solution do not necessarily exchange in the gas phase [23]. $\mathrm{H}_{6}$-Cex-cd has 581 exchangeable hydrogens. In the gas phase, +11 Cex ions exchange $23 \%$ of the exchangeable hydrogens, much lower than the $54 \%$ in solution. The noncovalent complexes exchange fewer hydrogens than the protein alone in solution, but more in the gas phase. This may be a result of the relay mechanism with $\mathrm{D}_{2} \mathrm{O}$ as a reagent in gasphase H/Dx [20]. For exchange, a hydrogen must be accessible on the surface of the protein, but also must be near a charged site, such as the N-terminal or charged side chains, such as Arg, His, and Lys. If binding the inhibitor leads to a more folded structure in the gas phase, as observed in the cross section measurements, some backbone amides on Cex may be closer to the charged sites, making H/Dx possible and leading to an increase in exchange rate as well as exchange level. With Cex alone, the hydrogens in these regions may be unable to complete H/D exchange because charged sites are farther from the exchangeable hydrogens. Increasing the charge on a complex or on Cex ions alone causes the exchange level to decrease, indicating that the addition of a single charge to the ions can change the conformations. This decrease in exchange level can also be explained with the relay mechanism. Ions with more charges generally have greater cross sections, corresponding to more unfolded conformations, which increase the distances between charged and exchange sites and results in a decrease in exchange levels [20].

Collision cross section and gas-phase H/Dx experiments provide insights into the gas-phase protein conformation. The cross section results illustrate that complex ions are more compact than free Cex ions on the ca. $1 \mathrm{~ms}$ time scale of the cross section measurements. The H/Dx results show that complex ions retain different and perhaps more folded conformations after $15 \mathrm{~s}$ of trapping at a pressure of $10 \mathrm{mTorr}$.

\section{Conclusion}

In this study, we have probed the conformational changes in enzyme noncovalently binding with small-molecule inhibitors. The gas-phase conformation and relative binding strengths of complexes are generally unchanged with sequence alterations far from binding sites. The relative gas-phase binding strengths measured by MS/MS follow the order, $\mathrm{X}_{2} \mathrm{IF}>\mathrm{X}_{2} \mathrm{IL}>\mathrm{X}_{2} \mathrm{DNJ}$, the same order as in solution.

Mass spectra of solutions containing the complexes show higher levels of free protein ions than expected. These Cex ions are mostly produced by dissociation of complex ions in the ion sampling process. In solution, Cex is almost entirely bound to inhibitors and, therefore, exchanges the same number of hydrogens as the complexes. In the gas phase, these Cex ions show cross sections similar to the complexes, but give H/Dx levels similar to Cex ions from a solution of Cex alone, suggesting these ions "remember" their original solution conformations on the ca. $1 \mathrm{~ms}$ time scale of the cross section experiments, but may fold or unfold on the seconds time scale of the H/Dx experiment.

Table 5. a. Numbers of Hydrogens Exchanged After $15 \mathrm{~s}$ of Gas-Phase H/Dx. b. Relative Exchange Levels

\begin{tabular}{llll}
$\mathrm{a}$ & & & \\
Ion & +11 & +12 & +13 \\
Cex-X $-\mathrm{X}_{2} \mathrm{DNJ}$ & $152 \pm 4$ & $141 \pm 2$ & $115 \pm 6$ \\
Cex-X $\mathrm{IL}$ & $140 \pm 3$ & $122 \pm 2$ & $112 \pm 6$ \\
Cex-X $\mathrm{IF}$ & $157 \pm 2$ & $148 \pm 2$ & $108 \pm 2$ \\
Cex & $131 \pm 4$ & $124 \pm 7$ & $109 \pm 5$ \\
$\mathrm{~b}$ & & & \\
Cex-X 2 DNJ & 1.00 & $0.93 \pm 0.03$ & $0.76 \pm 0.04$ \\
Cex-X $\mathrm{IL}$ & 1.00 & $0.87 \pm 0.02$ & $0.80 \pm 0.05$ \\
Cex-X $\mathrm{I}$ IF & 1.00 & $0.94 \pm 0.02$ & $0.67 \pm 0.01$ \\
Cex & 1.00 & $0.95 \pm 0.06$ & $0.83 \pm 0.05$ \\
\hline
\end{tabular}


The addition of a single charge is sufficient to change the gas-phase conformations of ions of the noncovalent complexes. As charge is added, cross sections of all the ions slightly increase, while H/Dx levels decrease. The cross sections of ions of the complexes decrease as the binding strengths increase. However the H/Dx levels of the gasphase complexes do not correlate with the binding strengths, also suggesting the ions may fold or unfold when trapped in the gas phase for several seconds.

In solution, complexes have lower H/Dx levels than Cex alone, consistent with previous NMR results [39] that show binding the inhibitors protects some hydrogens and reduces global fluctuations of the protein. In the gas phase, complex ions give smaller collision cross sections and interestingly greater $\mathrm{H} / \mathrm{Dx}$ levels than ions of Cex. The interpretation of the gas-phase H/Dx results requires considering two competing effects. To exchange, an exchangeable proton must be on the surface of the protein, favoring greater exchange levels for more unfolded proteins. However, an exchangeable hydrogen must also be near a charged site, favoring greater exchange levels of more folded ions of the same charge. Thus, H/Dx gives little insight into the degree of folding. However different H/Dx levels can indicate different conformations of a given ion. Cross sections show complexes have more compact conformations on the time scale of ca. $1 \mathrm{~ms}$. The H/Dx levels show at least the +11 and +12 complex ions retain different conformations from the Cex ions on the time scale of seconds.

The greater exchange levels of the noncovalent complexes are not inconsistent with complexes retaining more compact conformations of the time scale of seconds, but do not provide definitive evidence. In the future, pulsed timeresolved gas-phase H/Dx [45] might be used to study conformation changes of these or other trapped ions.

\section{Acknowledgements}

The authors acknowledge support for this project by the Natural Sciences and Engineering Research Council of Canada. They thank Stephen Withers' group for providing them with samples of enzyme and inhibitors.

\section{References}

1. Siuzdak, G.: The Expanding Role of Mass Spectrometry in Biotechnology, pp. 71-94. MCC Press, San Diego (2003)

2. Sinz, A.: Investigation of Protein-Ligand Interactions by Mass Spectrometry. Chem. Med. Chem. 2, 425-431 (2007)

3. Wyttenbach, T., Bowers, M.T.: Intermolecular Interactions in Biomolecular Systems Examined by Mass Spectrometry. Annu. Rev. Phys. Chem. 58, 511-533 (2007)

4. Clark, S.M., Konermann, L.: Determination of Ligand-Protein Dissociation Constants by Electrospray Mass Spectrometry-Based Diffusion Measurements. Anal. Chem 76, 7077-7083 (2004)

5. Sun, J., Kitova, E.N., Sun, N., Klassen, J.S.: Method for Identifying Nonspecific Protein-Protein Interactions in Nanoelectrospray Ionization Mass Spectrometry. Anal. Chem. 79, 8301-8311 (2007)

6. Kang, Y., Perrier, P., Douglas, D.J.: Mass Spectra and Ion Collision Cross Sections of Hemoglobin. J. Am. Soc. Mass Spectrom. 22, 290$299(2011)$
7. Hossain, B.M., Konermann, L.: Pulsed Hydrogen/Deuterium Exchange MS/MS for Studying the Relationship between Noncovalent Protein Complexes in Solution and in the Gas Phase after Electrospray Ionization. Anal. Chem. 78, 1613-1619 (2006)

8. Wang, W.J., Kitova, E.N., Sun, J.X., Klassen, J.S.: Blackbody Infrared Radiative Dissociation of Nonspecific Protein-Carbohydrate Complexes Produced by Nanoelectrospray Ionization: The Nature of the Noncovalent Interactions. J. Am. Soc. Mass Spectrom. 16, 1583-1594 (2005)

9. Barylyuk, K., Balabin, R.M., Grunstein, D., Kikkeri, R., Frankevich, V., Seeberger, P.H., Zenobi, R.: What Happens to Hydrophobic Interactions During Transfer from the Solution to the Gas Phase? The Case of Electrospray-Based Soft Ionization Methods. J. Am. Soc. Mass Spectrom. 22, 1167-1177 (2011)

10. Kang, Y., Douglas, D.J.: Gas-Phase Ions of Human Hemoglobin A, F and S. J. Am. Soc. Mass Spectrom. 22, 1187-1196 (2011)

11. Kitova, E.N., Bundle, D.R., Klassen, J.S.: Evidence for the Preservation of Specific Intermolecular Interactions in Gaseous Protein-Oligosaccharide Complexes. J. Am. Chem. Soc. 124, 9340-9341 (2002)

12. Kitova, E.N., Wang, W.J., Bundle, D.R., Klassen, J.S.: Retention of Bioactive Ligand Conformation in a Gaseous Protein-Trisaccharide Complex. J. Am. Chem. Soc. 124, 13980-13981 (2002)

13. Liu, L., Bagal, D., Kitova, E.N., Schnier, P.D., Klassen, J.S.: Hydrophobic Protein-Ligand Interactions Preserved in the Gas Phase. J. Am. Soc. Mass Spectrom. 131, 15980-15981 (2009)

14. Tesic, M., Wicki, J., Poon, D.K.Y., Withers, S.G., Douglas, D.J.: Gas Phase Noncovalent Protein Complexes that Retain Solution Binding Properties: Binding of Xylobiose Inhibitors to the $\beta-1,4$ Exoglucanase from Cellulomonas fimi. J. Am. Soc. Mass Spectrom. 18, 64-73 (2007)

15. Mark, K.J., Douglas, D.J.: Coulomb Effects in Binding of Heme in GasPhase Ions of Myoglobin. Rapid Commun. Mass Spectrom. 20, 111117 (2006)

16. Kaltashov, I.A., Eyles, S.J.: Studies of Biomolecular Conformations and Conformational Dynamics by Mass Spectrometry. Mass Spectrom. Rev. 21, 37-71 (2002)

17. Bai, Y.W., Milne, J.S., Mayne, L., Englander, S.W.: Primary Struture Effects on Peptide Group Hydrogen-Exchange. Proteins: Struct. Funct. Genet. 17, 75-86 (1993)

18. Cox, H.A., Julian, R.R., Lee, S.W., Beauchamp, J.L.: Gas-Phase H/D Exchange of Sodiated Glycine Oligomers with $\mathrm{ND}_{3}$ : Exchange Kinetics Do Not Reflect Parent Ion Structures. J. Am. Chem. Soc. 126, 64856490 (2004)

19. Valentine, S.J., Clemmer, D.E.: Temperature-Dependent H/D Exchange of Compact and Elongated Cytochrome $c$ Ions in the Gas Phase. J. Am. Soc. Mass Spectrom. 13, 506-517 (2002)

20. Wyttenbach, T., Bowers, M.T.: Gas Phase Conformations of Biological Molecules: The Hydrogen/Deuterium Exchange Mechanism. J. Am. Soc. Mass Spectrom. 10, 9-14 (1999)

21. Evans, S.E., Lueck, N., Marzluff, E.M.: Gas Phase Hydrogen/ Deuterium Exchange of Proteins in An Ion Trap Mass Spectrometer. Int. J. Mass Spectrom. 222, 175-187 (2003)

22. Freitas, M.A., Hendrickson, C.L., Emmett, M.R., Marshall, A.G.: GasPhase Bovine Ubiquitin Cation Conformations Resolved by Gas-Phase Hydrogen/Deuterium Exchange Rate and Extent. Int. J. Mass Spectrom. 187, 565-575 (1999)

23. Valentine, S.J., Clemmer, D.E.: H/D Exchange Levels of ShapeResolved Cytochrome $c$ Conformers in the Gas Phase. J. Am. Chem. Soc. 119, 3558-3566 (1997)

24. Mao, D.M., Ding, C.F., Douglas, D.J.: Hydrogen/Deuterium Exchange of Myoglobin Ions in a Linear Quadrupole Ion Trap. Rapid Commun. Mass Spectrom. 16, 1941-1945 (2002)

25. Campbell, S., Rodgers, M.T., Marzluff, E.M., Beauchamp, J.L.: Deuterium Exchange Reactions as a Probe of Biomolecule Structure. Fundamental Studies of Gas Phase H/D Exchange Reactions of Protonated Glycine Oligomers with $\mathrm{D}_{2} \mathrm{O}, \mathrm{CD}_{3} \mathrm{OD}, \mathrm{CD}_{3} \mathrm{CO}_{2} \mathrm{D}$, and $\mathrm{ND}_{3}$. J. Am. Chem. Soc. 117, 12840-12854 (1995)

26. Wright, P.J., Zhang, J.M., Douglas, D.J.: Conformations of Gas-Phase Ions of Ubiquitin, Cytochrome $c$, Apomyoglobin, and $\beta$-Lactoglobulin Produced from Two Different Solution Conformations. J. Am. Soc. Mass Spectrom. 19, 1906-1913 (2008)

27. Williams, S.J., Hoos, R., Withers, S.G.: Nanomolar Versus Millimolar Inhibition by Xylobiose-Derived Azasugars: Significant Differences between Two Structurally Distinct Xylanases. J. Am. Chem. Soc. 122, $2223-2235(2000)$ 
28. Notenboom, V., Williams, S.J., Hoos, R., Withers, S.G., Rose, D.R.: Detailed Structural Analysis of Glycosidase/Inhibitor Interactions: Complexes of Cex from Cellulomonas fimi with Xylobiose-Derived Aza-Sugars. Biochemistry 39, 11553-11563 (2000)

29. White, A., Withers, S.G., Gilkes, N.R., Rose, D.R.: Crystal-Structure of The Catalytic Domian of The $\beta$-1,4-Glycanase Cex from Cellulomonas fimi. Biochemistry 33, 12546-12552 (1994)

30. Wicki, J., Williams, S.J., Withers, S.G.: Transition-State Mimicry by Glycosidase Inhibitors: A Critical Kinetic Analysis. J. Am. Chem. Soc. 129, 4530-4531 (2007)

31. Chen, Y.L., Collings, B.A., Douglas, D.J.: Collision Cross Sections of Myoglobin and Cytochrome $c$ Ions with Ne, Ar, and Kr. J. Am. Soc. Mass Spectrom. 8, 681-687 (1997)

32. Glasoe, P.K., Long, F.A.: Use of Glass Electrodes to Measure Acidities in Deuterium Oxide. J. Phys. Chem. 64, 188-190 (1960)

33. Douglas, D.J., French, J.B.: Collisional Focusing Effects in RadioFrequency Quadrupoles. J. Am. Soc. Mass Spectrom. 3, 398-408 (1992)

34. Douglas, D.J.: An Aerodynamic Drag Model for Protein Ions. J. Am. Soc. Mass Spectrom. 5, 17-18 (1994)

35. Mao, D.M., Babu, K.R., Chen, Y.L., Douglas, D.J.: Conformations of Gas-phase Lysozyme Ions Produced from Two Different Solution Conformations. Anal. Chem. 75, 1325-1330 (2003)

36. Mauk, M.R., Mauk, A.G., Chen, Y.L., Douglas, D.J.: Tandem Mass Spectrometry of Protein-Protein Complexes: Cytochrome $c$-Cytochrome $b_{5}$. J. Am. Soc. Mass Spectrom. 13, 59-71 (2002)

37. Collings, B.A., Campbell, J.M., Mao, D.M., Douglas, D.J.: A Combined Linear Ion Trap Time-of-Flight System with Improved Performance and $\mathrm{MS}^{n}$ Capabilities. Rapid Commun. Mass Spectrom. 15, 1777-1795 (2001)
38. Mao, D.M., Douglas, D.J.: H/D Exchange of Gas Phase Bradykinin Ions in a Linear Quadrupole Ion Trap. J. Am. Soc. Mass Spectrom. 14, 85-94 (2003)

39. Poon, D.K.Y., Ludwiczek, M.L., Schubert, M., Kwan, E.M., Withers, S.G., McIntosh, L.P.: NMR Spectroscopic Characterization of a $\beta-(1,4)-$ Glycosidase Along Its Reaction Pathway: Stabilization upon Formation of the Glycosyl-Enzyme Intermediate. Biochemistry 46, 1759-1770 (2007)

40. White, A., Tull, D., Johns, K., Withers, S.G., Rose, D.R.: Crystallographic Observation of a Covalent Catalytic Intermediate in a $\beta$ Glycosidase. Nat. Struct. Biol. 3, 149-154 (1996)

41. Williams, S.J., Notenboom, V., Wicki, J., Rose, D.R., Withers, S.G.: A New, Simple, High-Affinity Glycosidase Inhibitor: Analysis of Binding through X-ray Crystallography, Mutagenesis, and Kinetic Analysis. J. Am. Chem. Soc. 122, 4229-4230 (2000)

42. Knapman, T.W., Morton, V.L., Stonehouse, N.J., Stockley, P.G., Ashcroft, A.E.: Determining the Topology of Virus Assembly Intermediates Using Ion Mobility Spectrometry-Mass Spectrometry. Rapid Commun. Mass Spectrom. 24, 3033-3042 (2010)

43. Creighton, T.E.: Proteins: Structure and Molecular Properties. W. H. Freeman and Company, New York (1993). 282

44. Poon, D.K.Y., Schubert, M., Au, J., Okon, M., Withers, S.G., McIntosh, L.P.: Unambiguous Determination of the Ionization State of a Glycoside Hydrolase Active Site Lysine by ${ }^{1} \mathrm{H}^{15} \mathrm{~N}$ Heteronuclear Correlation Spectroscopy. J. Am. Chem. Soc. 128, 15388-15389 (2006)

45. Wright, P. J., Douglas, D. J.: Time Resolved H/D Exchange of GasPhase Protein Ions in a Linear Ion Trap. Proceedings of the 54th ASMS Conference on Mass Spectrometry and Allied Topics; May 28-June 21, 2006, Seattle, WA. 